\title{
Aplicação da homeopatia na Odontologia: Uma revisão de literatura
}

\author{
Application of homeopathy in Dentistry: A literature review \\ Aplicación de la homeopatía en Odontología: Revisión de la literatura
}

Recebido: 17/06/2021 | Revisado: 25/06/2021 | Aceito: 26/06/2021 | Publicado: 11/07/2021

Sheyliane Rego Morais ORCID: https://orcid.org/0000-0002-7567-5892 Universidade Federal de Campina Grande, Brasil E-mail: sheylianerego@gmail.com

Filipe de Oliveira Lima ORCID: https://orcid.org/0000-0001-6652-3101 Universidade Federal de Campina Grande, Brasil E-mail: filipelimaoliveirava@outlook.com

Ana Beatriz Rodrigues Moura ORCID: https://orcid.org/0000-0003-0006-148X Universidade Federal de Campina Grande, Brasil E-mail: ana_beatriz_882@hotmail.com

Natália Oliveira Matos

ORCID: https://orcid.org/0000-0001-6590-6901 Universidade Federal de Campina Grande, Brasil E-mail: nataliaomatos@gmail.com

Rafaella Bandeira de Melo Souza Cavalcanti ORCID: https://orcid.org/0000-0001-8765-2447 Universidade Federal de Campina Grande, Brasil E-mail: Rafaella.bm@hotmail.com

Fabiana Larissa Santos de Medeiros ORCID: https://orcid.org/0000-0002-3804-600X Universidade Federal de Campina Grande, Brasil E-mail: fabiana.serido@hotmail.com

Rodrigo Machado Castro

ORCID: https://orcid.org/0000-0002-5299-2094 Universidade Federal de Campina Grande, Brasil

E-mail: castrorm09@gmail.com

Rosana Marques da Silva

ORCID: https://orcid.org/0000-0002-5947-1996 Universidade Federal de Campina Grande, Brasil E-mail: rosanamarques358@gmail.com

Vitor Nascimento Goes

ORCID: https://orcid.org/0000-0001-5163-1126 Universidade Federal de Campina Grande, Brasil E-mail: vitor9511goes@hotmail.com Nathan Felipe de Brito Lima

ORCID: https://orcid.org/0000-0001-5259-0717 Universidade Federal da Paraíba, Brasil

E-mail: nathanodontologia@gmail.com

Maria Angélica Sátyro Gomes Alves ORCID: https://orcid.org/0000-0003-3329-8360 Universidade Federal de Campina Grande, Brasil E-mail: angelicasatyro@hotmail.com

Luanna Abílio Diniz Melquíades de Medeiros ORCID: https://orcid.org/0000-0002-1630-3968 Universidade Federal de Campina Grande, Brasil

E-mail: luannaabiliod@gmail.com

Gymenna Maria Tenório Guênes ORCID: https://orcid.org/0000-0002-5447-0193 Universidade Federal de Campina Grande, Brasil

E-mail: gymennat@yahoo.com.br 


\begin{abstract}
Resumo
A Homeopatia é uma ciência que busca compreender o processo saúde-doença, através da perspectiva do equilíbrio e desequilíbrio do organismo, e vê-lo como um todo. Possui quatro princípios básicos, "Cura pela similitude", "Experimentação de medicamentos em indivíduos sadios", "Uso de medicamentos dinamizados" e "Prescrição de medicamentos individualizados". E mesmo sendo uma terapia reconhecida, muitos profissionais da Odontologia ainda desconhecem essa ciência. Dessa forma o objetivo deste trabalho foi realizar uma revisão de literatura abordando a aplicação da Homeopatia na Odontologia. Foi desenvolvida uma revisão de literatura narrativa com o tema Homeopatia e sua aplicabilidade na Odontologia, a qual foi baseada em trabalhos dos anos 2010 à 2020. Encontrou-se para periodontia um bom efeito redutor de características da periodontite crônica, principalmente quando correlacionado a terapia convencional; na Odontopediatria os estudos mostram o efeito clínico, podendo inclusive ser associado a outras práticas que focam no emocional da criança. Já na área cirúrgica alguns estudos mostraram a eficácia de medicamentos Homeopáticos para edema em comparação à medicamentos alopáticos. Assim, pode-se perceber que já existem estudos que comprovam os benefícios que os medicamentos Homeopáticos proporcionam à prática odontológica, mostrando-os como alternativas à medicina convencional, no entanto alguns estudos experimentais necessitam de mais fases para comprovar os seus efeitos.
\end{abstract}

Palavras-chave: Homeopatia; Odontologia; Práticas integrativas e complementares.

\begin{abstract}
Homeopathy is a science that seeks to understand the health-disease process, through the perspective of the organism's balance and imbalance, and to see it as a whole. It has four basic principles, "Cure for similarity", "Experimenting with medications in healthy individuals", "Use of dynamized medications" and "Prescription of individual medications". And even though it is a recognized therapy, many dental professionals are still unaware of this science. Thus, the objective of this work was to carry out a literature review addressing the application of Homeopathy in Dentistry. A narrative literature review was developed with the theme Homeopathy and its applicability in Dentistry, which was based on works from the years 2010 to 2020. A good reducing effect of chronic periodontitis characteristics was found for periodontics, especially when correlated to conventional therapy; in Pediatric Dentistry, studies show the clinical effect, and may even be associated with other practices that focus on the child's emotional. In the surgical area, some studies have shown the effectiveness of Homeopathic medicines for edema compared to allopathic medicines. Thus, it can be seen that there are already studies that prove the benefits that Homeopathic medicines provide to dental practice, showing them as alternatives to conventional medicine, however, some experimental studies need more phases to prove their effects.
\end{abstract}

Keywords: Homeopathy; Dentistry; Integrative and complementary practices.

\title{
Resumen
}

La homeopatía es una ciencia que busca comprender el proceso salud-enfermedad, a través de la perspectiva del equilibrio y desequilibrio del organismo, y verlo como un todo. Tiene cuatro principios básicos, "Curar la similitud", "Experimentar con medicamentos en individuos sanos", "Uso de medicamentos dinamizados" y "Prescripción de medicamentos individualizados". Y aunque es una terapia reconocida, muchos profesionales de la odontología aún desconocen esta ciencia. Así, el objetivo de este trabajo fue realizar una revisión de la literatura que aborde la aplicación de la Homeopatía en Odontología. Se desarrolló una revisión de literatura narrativa con el tema Homeopatía y su aplicabilidad en Odontología, la cual se basó en trabajos de los años 2010 a 2020. Se encontró un buen efecto reductor de las características de la periodontitis para la periodoncia, principalmente cuando se correlaciona con terapia convencional; en Odontopediatría, los estudios muestran el efecto clínico, e incluso pueden estar asociados con otras prácticas que se enfocan en la emocionalidad del niño. En el área quirúrgica, algunos estudios han demostrado la eficacia de los medicamentos homeopáticos para el edema en comparación con los medicamentos alopáticos. Así, se puede apreciar que ya existen estudios que prueban los beneficios que brindan los medicamentos homeopáticos a la práctica odontológica, mostrándolos como alternativas a la medicina convencional, sin embargo algunos estudios experimentales necesitan más fases para comprobar sus efectos.

Palabras clave: Homeopatía; Odontología; Prácticas integradoras y complementarias. 


\section{Introdução}

A Organização Mundial de Saúde (OMS) se refere as práticas médicas originárias culturalmente em cada país como Medicina Tradicional, classificada em Medicinas Tradicionais/Complementares e Alternativas. No Brasil foram implementadas em esfera nacional somente em 2006, e foram denominadas como Práticas Complementares e Integrativas (PICS), que são a união de ações terapêuticas, que se distinguem da biomedicina ocidental, pois utilizam ervas, vegetais e fragmentos de animais, incluem atividades manuais, espirituais e corporais, além de não utilizar medicamentos quimicamente purificados (Fontanella, 2007; Sousa et al., 2012).

Atualmente o Sistema Único de Saúde (SUS) disponibiliza 29 PICS que visam expandir a visão sobre o processo saúde-doença, promover o desenvolvimento terapêutico, a integração para com o meio ambiente e comunidade. Mas somente Acupuntura, Fitoterapia, Homeopatia, Hipnose, Laserterapia, Terapia Floral, Medicina antroposófica e a Ozonioterapia são regulamentadas e reconhecidas pelo Conselho Federal de Odontologia (CFO), tornando assim os Cirurgiões - Dentistas aptos a praticá-las, possibilitando um tratamento mais individual, focando nas particularidades de cada indivíduo e sua doença (Bueno, 2019; Gonçalves et al., 2018).

Uma dessas práticas é a Homeopatia, uma ciência médica originada há mais de 200 anos, fundamentada pelo médico alemão Christian Frederich Samuel Hahnemann, a qual se baseia em quatro pilares: "Princípio de cura pela similitude", "Experimentação de medicamentos em indivíduos sadios", "Uso de medicamentos dinamizados" e "Prescrição de medicamentos individualizados". No Brasil esse conhecimento se propagou após a oficialização do seu ensino (Santos \& Sá, 2014; Teixeira, 2013).

$\mathrm{Na}$ atualidade, cada vez mais aumentam a procura por práticas alternativas em saúde, e na consulta odontológica não é diferente, os pacientes têm buscado tratamentos diferenciados, que os valorizem individualmente e que se preocupem em promover seu bem-estar geral. A Homeopatia se mostra como um tratamento onde o profissional define um diagnóstico mais amplo do processo patológico, possibilitando assim aplicar recursos terapêuticos homeopáticos comprovados na área odontológica, substituindo medicações alopáticas, que podem provocar danos indesejáveis, como o aumento da resistência aos antibióticos (Costa et al., 2016; Eleutério; Oliveira \& Pereira Júnior, 2011).

Percebendo a importância das práticas integrativas complementares na Odontologia, especialmente a Homeopatia, torna-se justificável uma revisão de literatura abordando o direcionamento da mesma para pacientes odontológicos a fim de se reunir comprovações cientificas e conhecimento sobre os benefícios que podem agregar.

\section{Metodologia}

O método utilizado para o desenvolvimento deste trabalho foi uma revisão de literatura narrativa com o tema Homeopatia e sua aplicabilidade na Odontopediatria, Cirurgia Bucomaxilofacial e Periodontia, baseada em trabalhos dos anos 2010 à 2020. Esse tipo de metodologia possui uma temática mais aberta, sem um protocolo específico, mas elaborada a partir de artigos relevantes, selecionados de maneira livre (Cordeiro et al., 2007).

Foram consultados os bancos de dados Bireme, Lilacs, Medline e Pubmed, empregando como palavras-chave: Homeopatia, Odontologia, Cirurgia bucomaxilofacial, Periodontia, Odontopediatria e Práticas integrativas e complementares.

\section{Resultados e Discussão}

A Homeopatia é considerada uma modalidade terapêutica complexa, que prega que todo indivíduo além de seu ser físico possui um mecanismo imaterial, e que o adoecimento é o processo de desequilíbrio entre o que é considerado uma adaptação e compensação normal e manifestações subjetivas como emoções, pensamentos, vontades, aversões, relação com o 
sono, como também outros aspectos clínicos habituais, dessa forma pode-se obter um diagnóstico para o estado do ser, e realizar a prescrição do medicamento homeopático segundo seus princípios (Hanhmann, 1996; Teixeira, 2007).

A utilização de sedação via oral para realização do atendimento odontológico, principalmente para pacientes infantis não é muito difundida no Brasil, pois existem outras maneiras de realizar o controle da ansiedade desse tipo de paciente sejam farmacológicas ou não (Ferreira Junior et al., 2010).

Dentre as possibilidades de controle da ansiedade, as terapias complementares e alternativas, como é o exemplo da Homeopatia podem trazer benefícios à diversos procedimentos e áreas da odontologia, associada a capacitação do cirurgiãodentista, podendo também minimizar estados psicológicos de pacientes infantis no caso da odontopediatria. Esse especialista pode utilizar do mecanismo homeopático, se for o caso, para auxiliar no pré-operatório de crianças que apresentam transtornos como medo e ansiedade pelo tratamento. Mesmo que não tenham comprovações cientificas suficientes afirmando que esses medicamentos reduzem os níveis desses transtornos, também não há estudos que os neguem, portanto é importante aumentar a inter-relação entre essa ciência e demais áreas da Odontologia, a fim de encontrar comprovações e novas perspectivas cientificas principalmente direcionadas a odontopediatria (Eleutério; Oliveira \& Pereira, 2011).

Um estudo de Giorgi et al. (2010), com 48 pacientes comparou o efeito de um fármaco homeopático e um medicamento a base de benzodiazepínicos. Os indivíduos foram separados em três grupos; ao primeiro foi administrado a terapêutica homeopática, ao segundo grupo Diazepam, e ao terceiro que era o grupo controle, não foi prescrito nada. Após o período de 3 meses pode-se perceber que em todos os grupos houve redução da ansiedade, porém no grupo de tratamento homeopata o nível de redução foi o maior, mostrando a sua potência em relação aos outros. Além disso, 23\% dos pacientes tratados alopaticamente apresentaram efeitos adversos, já no primeiro não se observou nenhum caso. Sinalizando, portanto, sua possível utilização para esta finalidade, em situações cabíveis, ainda que estudos testes em pacientes nessa faixa etária sejam necessários.

Côrrea (2010) por exemplo, enaltece que o tratamento alternativo homeopático é capaz de trazer benefícios as crianças com bruxismo, já que muitas vezes o principal fator etiológico é a ansiedade, e visa através desse, cuidar do lado emocional, podendo ser associado aos florais de Bach e acupuntura.

Já Valença (2014) realizou um estudo direcionando essa prática integrativa na sintomatologia da erupção dentária decídua, que pode ser leve ou se exacerbar causando sintomas como febre alta e/ou vômito, dentre outros. Os principais medicamentos prescritos nesse tipo de tratamento foram: Aconitumnapellus usado para febre e dor intensa, Belladonna, Bórax, Calcarea carbônica, Calcarea phosphorica, Chamomilla, Magnesia phosphorica, Kreosotum, Phytolacca decandra, Silicea, que tratam dor, febre, inflamação das gengivas, diarréia, secreção, dentre outros sintomas seguindo os princípios do método. Muitos profissionais da área acreditam que essas alterações são causadas pela erupção e devem ser tratados os sintomas característicos dessa fase, portanto cada caso tem que ser individualizado e seguidas as orientações de acordo com o profissional capacitado, a fim de recomendar o medicamento homeopático ideal.

Estudando os sinais e sintomas de estomatites virais em pacientes infantis, Greck (2016) buscou um medicamento homeopático que pudesse ter aplicabilidade sobre esse tipo de lesão, que dentre os principais sintomas orais apresentou: ardência, fácil sangramento, dor em diversas áreas da cavidade bucal e gânglios inchados. Seguindo o preceito de que o medicamento deve ser escolhido de acordo com a totalidade dos sintomas apresentados pelo indivíduo, o que apresentou o melhor desempenho foi o Borax (Biborato de Sódio), que agiu aliviando a irritação, tratando e levando a cura.

Prata Júnior et al. (2019), relataram o caso de uma paciente infantil com diagnóstico de Lesão Central de Células Gigantes, que realizou a exérese da lesão através da curetagem com solução de Carnoy. Após o período de proservação e percepção de imagem radiopaca, optou-se pelo tratamento concomitante com terapia homeopática utilizando Carcinosinum C30, dez glóbulos em jejum e antes de dormir; e Symphytum C6 + Calcária Carbônica C6, dez glóbulos quatro vezes ao dia; e 
duas aplicações intralesionais de $0,5 \mathrm{~mL}$ de corticóide (Triancinolona hexacetonida $20 \mathrm{mg} / \mathrm{mL}$ ), com intervalo de 1 mês entre uma aplicação e outra. Após um mês da última aplicação, percebeu-se através de exames imaginológicos a neoformação óssea em algumas regiões. $\mathrm{O}$ uso de Homeopatia foi contínuo por ainda 3 meses e após 4 meses do protocolo confirmou-se de fato a neoformação óssea no local da lesão.

A literatura também relata a aplicabilidade da Homeopatia na Periodontia. Silva, Fischer e Terezan (2010) relatam clinicamente que obtiveram êxito ao relacionar um tratamento periodontal em paciente com periodontite crônica, e terapia homeopática complementar de acordo com seus princípios. Os sintomas que guiaram a escolha do medicamento homeopático Mutus (Lachesis mutus) foram principalmente mentais, pois segundo o trabalho, os sintomas periodontais se mostraram como uma consequência do desequilíbrio vital. É importante ressaltar que mesmo após três anos de terapia periodontal de suporte a paciente não tinha apresentado resultados satisfatórios, no entanto após a concomitante co-relação dos tratamentos houve significativa redução das Profundidades de Sondagens de Bolsas (PSB), do Índice de Placa (IP) e do Percentual de Sangramento à Sondagem (SS) ao longo do tempo.

Mourão, Moutinho e Canabarro (2013) também realizaram estudos em pacientes com periodontite crônica, utilizando medicamentos homeopáticos a fim de avaliar a adição de benefícios, usando-o como adjuvante ao tratamento periodontal convencional não cirúrgico. Os medicamentos utilizados foram: Berberis $6 \mathrm{CH}$ (dose de dois comprimidos, duas vezes ao dia, 45 dias), Mercurius solubilis / Belladona / Hepar enxofre e 6 CH (dose de dois comprimidos, três vezes ao dia) durante 15 dias, e Nosódios e Pirogênio200 CH (dose semanal, por 2 semanas). A amostra contou com 60 indivíduos de ambos os sexos, sendo 20 saudáveis e 40 com periodontite, estes foram divididos ainda em 2 grupos iguais (1 controle: tratados apenas com tratamento periodontal convencional, e 1 grupo teste: tratados com associação dos métodos. No início do estudo os pacientes com periodontite possuíam valores mais altos de Colesterol LDL e na glicose, do que o grupo com pacientes saudáveis. Após 90 dias, percebeu-se que os pacientes afetados pela periodontite haviam reduzido os níveis sistêmicos (Colesterol Total, Triglicerídeos, Glicose e Ácido Úrico), com exceção do LDL e HDL no grupo de tratamento convencional, e exceto HDL no grupo teste. Além disso houve um aumento estatístico no nível de inserção clínica (+ 0,51mm) no grupo tratado também com a homeopatia. Além de redução de profundidade e de sangramento à sondagem, e do nível de placa visível em ambos os grupos que possuíam periodontite.

Esse grupo de pacientes também foi estudado por Mourão (2015) que avaliou os efeitos da Homeopatia na periodontite crônica generalizada em pacientes que possuem diabetes tipo II. Tratou-se de um ensaio clínico randomizado, duplo-cego, de 1 ano de duração. Participaram do estudo 80 pacientes de ambos os sexos, afetados pela periodontite e diabetes, com idades entre 32 e 70 anos, foram divididos ainda em dois grupos iguais, grupo teste que recebeu placebo e o grupo tratado com os medicamentos Berberis, Mercurius solubilis/ Belladona/ Hepar sulfure e Pyrogenium. No entanto os dois grupos foram tratados com terapia periodontal convencional. Avaliou-se após um período de 30 dias, 6 e 12 meses, através dos parâmetros clínicos e laboratoriais os efeitos locais e sistêmicos, além de um questionário de qualidade de vida. Observou-se que no grupo em que foi realizado o tratamento homeopático como coadjuvante os níveis de LDL, colesterol total, hemoglobina glicada, glicemia, e ácido úrico foram reduzidos. Ambos os grupos mostraram resultados clínicos favoráveis ao controle da periodontite ao final do estudo, no entanto os resultados do grupo tratado com Homeopatia indicam que além de reduzir os níveis laboratoriais, são capazes de promover uma melhor qualidade de vida em pacientes com essas alterações.

Cabresté (2015) desenvolveu um estudo comparativo entre o Nimesulida, a Arnica montana fitoterápica, e a Arnica montana 6 CH homeopática, para verificar seu aproveitamento em clínica odontológica e no tratamento de doença periodontal experimental induzida em ratos por ligadura. A administração dos medicamentos era feita diariamente, e $\mathrm{H}_{2} \mathrm{O}$ (grupo controle), em quatro grupos de ratos, durante 14, 21 e 28 dias. Concluiu-se que ambas as Arnicas sinalizam que podem ser benéficas na 
terapia periodontal experimental em questão, e que pode ter aplicabilidade em tal patologia, principalmente frente aos efeitos adversos do Nimesulida. Trata-se de uma pesquisar inovadora, no entanto, é necessário aprofundar com estudos em mais fases.

A Arnica homeopática também foi estudada por Kawakami et al., (2011) em cujo trabalho se mostrou capaz de modular o processo de inflamação aguda em ratos, já que age sobre prostaglandinas e histamina, que são mediadores do processo de formação do edema.

Já Giorgi (2011) relata o caso de uma paciente que desenvolveu reabsorção radicular severa nos elementos 11, 12, 21 e 22, em consequência de fatores causados pelo tratamento ortodôntico, e áreas de reabsorção óssea causadas por doença periodontal. Em razão da negação da paciente em realizar as exodontias de tais elementos, optou-se, portanto, pela Homeopatia como tratamento, utilizando simillimum Aconitum napellus (líquido), com diluição $12 \mathrm{CH}$ uma vez ao dia, durante trinta dias. Após seis meses de tratamento percebeu-se através do exame radiográfico que houve a estabilização da reabsorção radicular. De acordo com o relato, o caso teve seguimento de dezoito meses sendo a paciente medicada nas diluições de $12 \mathrm{CH}, 18 \mathrm{CH}$, e $30 \mathrm{CH}$.

Souza et al. (2011), realizaram uma pesquisa experimental, randomizada, duplo-cega, comparativa entre a Arnica montana $6 \mathrm{CH}$, e o Diclofenaco de Sódio $50 \mathrm{mg}$, a fim de verificar a atividade anti-edematosa frente a exodontias de terceiros molares inclusos. Contou-se com 30 voluntários, que possuíam o terceiro molar incluso bilateralmente, e que foram removidos com técnica cirúrgica padronizada e pelo mesmo operador. Dividiu-se então em dois, onde o Grupo A fez uso do medicamento homeopático, cinco dias antes da realização do procedimento e durante oito dias após, seis gotas, quatro vezes ao dia, e o Grupo D fez uso do AINE a cada oito horas, durante três dias após o procedimento. A mensuração do edema foi feita quatro vezes para ambas as técnicas após os mesmos períodos, e a partir do: 1) Canto do olho à região do trágus, 2) canto do olho ao ângulo mandibular, 3) comissura labial ao trágus. Percebeu-se que o medicamento homeopático teve uma boa atividade no controle do edema, similar ao efeito do outro grupo. De acordo com o estudo, $65 \%$ dos pacientes afirmaram que tiveram um pós operatório mais confortável quando tratados com a Homeopatia, em comparação com o outro grupo. Indicando assim esta PICS como uma alternativa aos medicamentos alopáticos e para os pacientes impossibilitados de fazer uso do Diclofenaco de Sódio $50 \mathrm{mg}$.

Souza (2018) desenvolveu um estudo clínico randomizado, triplo-cego, com metodologia para avaliar a atividade preemptiva do Traumeel $S \circledR$ em comparação com Dexametasona, em relação a parâmetros como edema, dor e trismo em exodontias de terceiros molares inferiores. Realizou-se o procedimento em 17 voluntários recebiam medicação preemptiva intramuscular no masseter com 15 dias de intervalo entre um processo de intervenção com um medicamento e outro. Para análise da percepção dos indivíduos foram avaliados clinicamente após 24,48 e 72 horas e 7 dias, e os fatores foram mensurados através da medida da face para edema, abertura máxima bucal para trismo, e já para dor usou-se a Escala Visual Analógica (EVA) que avalia a intensidade da dor sentida pelo paciente. Como resultados percebeu-se que para edema não houve diferença estatística relevante entre ambos, já para dor e trismo a Dexametasona se mostrou mais eficaz, nos períodos de 48, e 24-48h respectivamente em relação ao Traumeel $S \circledR$.

Sales et al, (2014), avaliaram o efeito benéfico do tratamento homeopático em exodontias que tiveram intercorrências transoperatórias como fratura de raiz, de septo interdental, de cortical, de túber, comunicação bucosinusal. Os 48 pacientes foram divididos aleatoriamente em dois grupos. Em um grupo foi utilizado o medicamento homeopático AlivioHo-trauma posologia de cinco gotas sublinguais, três vezes sequenciadas com intervalos de 15 minutos, por três vezes ao dia, até que os sintomas desaparecessem. E o grupo controle utilizou tratamento alopático com os medicamentos convencionais para cada caso. Percebeu-se que o medicamento homeopático teve eficácia semelhante ao grupo controle para dor e inflamação e nenhuma reação adversa foi sugerida. E em ambos os tratamentos não houve presença de alveolite. 
O potencial radioprotetor também foi avaliado por Nery (2011) que estudou um medicamento homeopático feito à base de radiação x e uma formulação fitoterápica contendo óleo de copaíba versus a vitamina E. Foram estudados 200 ratos divididos em grupos. No grupo 1 (controle), foi utilizado $\mathrm{NaCl}$ 0,9\% e não houve radiação; o $\mathrm{G} 2$ recebeu o mesmo porém com radiação; no G3 foi utilizado suspensão de acetato de dl-alfa-tocoferol (vitamina E) 40mg/kg/vo/dia; o G4 recebeu o mesmo tratamento do grupo anterior, mas com radiação; o G5 foi o grupo que recebeu o protocolo homeopático, com radiação $\mathrm{x}$ dinamizada a $15 \mathrm{CH} 1 \mathrm{~mL} / \mathrm{kg} / \mathrm{vo} / \mathrm{dia}$; o grupo 6 recebeu a mesma fórmula homeopática, com adição de irradiação; o G7 recebeu a formulação fitoterápica, com óleo de copaíba $2 \mathrm{~g} / \mathrm{kg} / \mathrm{vo} / \mathrm{dia}$ e o último grupo (G8) recebeu o mesmo do anterior, porém com irradiação. Todos os grupos receberam o protocolo por 7 dias, e nos grupos que sofreram radiação, os animais receberam medicação por mais 7 dias. Após certos períodos de tempo, utilizou-se pilocarpina para estimular a saliva para a coleta. Foi observado a função salivar e a morfologia da parótida. Nenhum dos tratamentos afetou significativamente a produção de saliva. No entanto, percebeu-se que nenhuma das formulações teve potencial radioprotetor para as glândulas e para a função salivar.

Alencar (2013) realizou uma avaliação do efeito radioprotetor em ratos que passaram pelo procedimento de radiação. O estudo foi realizado com 150 animais, divididos em 6 grupos. O grupo 1 recebeu solução salina (controle) e não foi submetido a radiação; o grupo 2 recebeu o mesmo tratamento, mas com radiação de $15 \mathrm{~Gy}$; com o grupo 3 utilizou-se solução hidroalcoólica dinamizada em $15 \mathrm{CH}$, o grupo 4 foi submetido ao mesmo tratamento do grupo anterior, porém com dose de radiação; o grupo 5 recebeu o tratamento homeopático, com $0,25 \mathrm{ml}$ de solução hidroalcóolica com radiação de $15 \mathrm{~Gy}$ e $15 \mathrm{CH}$ dinamizada; o último grupo recebeu o mesmo tratamento do grupo 5, mas com radiação. Após 12 horas, 3, 10, 17 e 24 dias, induziu-se a salivação dos animais. Pode-se verificar que com 17 dias, os animais submetidos a radiação e tratados com Homeopatia tiveram maior índice de salivação que os demais na mesma situação. Realizou-se também a análise morfométrica do número de ácinos em relação ao tempo, observou-se que os animais que receberam álcool e foram irradiados tiveram uma redução do número de ácinos ao longo do tempo. Concluiu-se que o tratamento homeopático provocou um efeito radioprotetor, ainda que tardio, para a função salivar e glândula parótida.

\section{Considerações Finais}

Baseado nas informações coletadas na literatura, pode-se perceber que a Homeopatia é uma ciência capaz de trazer inúmeros benefícios aos pacientes, nas referidas áreas da Odontologia, se aplicada corretamente, e de acordo com seus princípios. Torna-se ainda uma grande alternativa e/ou complemento para a medicina convencional, já que nos estudos relatados não há indícios de efeitos adversos pela mesma. No entanto mais estudos são necessários para a comprovação do efeito de alguns medicamentos homeopáticos, principalmente mais fases para os estudos ainda experimentais

\section{Referências}

Alencar, P. N. B. (2013). Efeito da homeopatia na função salivar e na morfologia de glândulas parótidas de ratos irradiados. [Dissertação de mestrado, Universidade Estadual de Campinas].

Bueno, N., Fernandes, A. P., Martorell, L. B., Franco, L. L. M. M., \& Reis, L. B. M. (2019). Práticas integrativas e complementares: implantação nos serviços público e privado de saúde na odontologia. Anais da Jornada Odontológica de Anápolis-JOA. http://45.4.96.34/index.php/joa/article/view/4202/2573.

Cabresté, A. (2015). Estudo comparativo entre as ações do nimesulida, arnica montana homeopática e arnica montana fitoterápica-possíveis aplicações na terapêutica da doença periodontal e na rotina do consultório odontológico. [Tese de Doutorado. Universidade de São Paulo].

Cordeiro, A. M., Oliveira, G. M., Renteria, J. M., \& Guimarães, C. A. (2007). Revisão sistemática: Uma revisão narrativa. Rev. Col. Bras. Cir. 34 (6), 428431.

Corrêa, M. S. N. P. (3a ed). (2010). Odontopediatria na Primeira Infância. Quintessence. 
Costa, R. F., Volpato, S., Gallon, A., \& Dirschnabel, A. J. (2016). Biocampo Energético: Atuação da Homeopatia na Odontologia. Unoesc. \& Ciência-ACBS, 7 (2),137-144.

Eleutério, A. S. L., Oliveira, D. S. B., \& Pereira Júnior, E. S. (2011). Homeopatia no controle do medo e ansiedade ao tratamento odontológico infantil: revisão. Rev. odontol. Univ. Cid. São Paulo (online),23 (3), 238-244.

Ferreira Junior F. O. M., Mundim A. P., Gomes H. S., Sampaio F. C., Machado G. C. M., \& Costa P. S. S. (2010). Uso de sedação oral para o atendimento odontológico no neso. https://files.cercomp.ufg.br/weby/up/399/o/OSMAR_MARTINS_FERREIRA_JUNIOR.pdf.

Fontanella, F., Speck, F. P., Piovezan, A. P., \& Kulkamp, I. C. (2007). Conhecimento, acesso e aceitação das práticas integrativas e complementares em saúde por uma comunidade usuária do Sistema Único de Saúde na cidade de Tubarão/SC. Arquivos catarinenses de Medicina, 36 (2), 69-74.

Greck, A. P. B. S.(2016). A homeopatia nas estomatites virais infantis: preliminar para o gênio epidêmico. [Tese de Monografia, Escola Bahiana de Medicina e Saúde Pública].

Giorgi, M. S., Borelli Neto, L., Frias, A. C., Santos, C. M. S., \& Trindade, I. (2010). Contribuição da homeopatia no controle da ansiedade e do medo como prevenção das emergências médicas em odontologia. Revista de Homeopatia, 73(3/4), 17-22.

Giorgi, M. S. (2011). Avaliação do medicamento homeopático no tratamento da reabsorção radicular severa: relato de caso. Revista de Homeopatia, 74(3).

Gonçalves, R. N., Gonçalves, J. R. S. N., Buffon, M. C. M., Negrelle, R. R. B., \& Albuquerque, G. S. C. (2018) Práticas Integrativas e Complementares: inserção no contexto do ensino Odontológico. Revista da ABENO, 18 (2), 114-123,

Hahnemann, S. (1996). Materia medica pura (Vol. 2). B. Jain Publishers.

Kawakami, A. P., Sato, C., Cardoso, T. N., \&Bonamin, L. V. (2011). Inflammatory process modulation by homeopathic Arnica montana $6 \mathrm{CH}$ : the role of individual variation. Evidence-based complementary and alternative medicine.

Mourão, L. C., Moutinho, H., \& Canabarro, A. (2013). Additional benefits of homeopathy in the treatment of chronic periodontitis: a randomized clinical trial. Complementary Therapies In Clinical Practice, 19 (4), 246-250.

Mourão, L. C. S. (2015). Efeitos da homeopatia no tratamento da Periodontite Crônica Generalizada em pacientes diabéticos tipo II: ensaio clínico randomizado, duplo-cego, placebo-controlado, com um ano de acompanhamento. [Tese de Doutorado, Universidade Estadual do Rio de Janeiro]. http://www.bdtd.uerj.br/tde_busca/arquivo.php?codArquivo=13884 .

Nery, L. R. (2011). Efeito da homeopatia, fitoterapia e vitamina E sobre glândula parótida de ratos irradiados. [Tese de doutorado, Universidade Estadual de Campinas].

Prata Júnior, A. G., França, A. B., Silva, A. G. S., Trento, C. L., Souza, L. M. A., \& Takeshita, W. M. (2019). Tumor central de células gigantes en paciente pediátrico: exéresis, reabordaje y homeopatía. Rev Cubana Estomatol. 56 (4), 1-12.

Sales, K. C. C., Sánchez, E. Z., Iglesias, R. A., Méndez, H. T. D., \& Pérez, P. D. A. (2014). Efectividad de la homeopatia em el tratamiento de exodoncias traumáticas. Revista Electrónica Dr. Zoilo E. Marinello Vidaurreta, 39(12).

Santos, R., \& Sá, F. M. P. (2014). Homeopatia: histórico e fundamentos. Revista Científica da Faculdade de Educação e Meio Ambiente, 5 (1), $60-78$.

Silva, E. B., Fischer, R. G., \& Terezan, M. L. F. (2010). Homeopatia como coadjuvante na terapia periodontal de suporte em paciente com periodontite crônica: relato de caso clínico. R. Periodontia, 37-41.

Sousa, I. M. C., Bodstein, R. C. A., Tesser, C. D., Santos, F. A. Z., \& Hortale, V. A. (2012). Práticas integrativas e complementares: oferta e produção de atendimentos no SUS e em municípios selecionados. Cadernos de Saúde Pública, 28, 2143-2154.

Souza, G. M. (2018). Administração preemptiva do Traumeel $S \circledR$ versus Dexametasona em cirurgias de terceiros molares inferiores: ensaio clínico randomizado, triplo-cego. [Tese de Mestrado, Universidade Federal dos Vales do Jequitinhonha e Mucuri].

Souza, L. M. A., Dantas, A. L. L., Ribeiro, A. O., Ramacciato, J. C., \& Motta, R. H. L. (2011). Ação Anti-Edematosa: Arnica montana 6ch X Diclofenaco de Sódio 50 mg. Pesquisa Brasileira em Odontopediatria e Clínica Integrada, 11(4), 491-496.

Teixeira, M. Z. (2013). Similia similibus curentur: o princípio de cura homeopático fundamentado na farmacologia moderna. Revista de Medicina, 92 (3),183203.

Teixeira, M. Z. (2007). Homeopatia: prática médica coadjuvante. Revista da Associação Médica Brasileira, 53(4), 374-376.

Valença, R. M. (2014). Tratamento Homeopático na erupção dentária decídua. [Tese de Monografia, Centro Alpha de Ensino]. 